responsiveness: a dynamic interplay between genes, cells and molecules'. The symposium must have been an exciting occasion held in a city which has contributed much to the evolution of immunological thought. The authors have produced a number of interesting and sometimes provocative chapters, and the whole volume makes interesting reading. However, for the medical geneticist, Essential Immunology by Roitt, now in its 3rd edition, provides a more comprehensive and systematic study of immunology, while the eagerly awaited 2nd edition of Fudenberg's Basic Immunogenetics is soon to be published by Oxford University Press.

Perhaps one might paraphrase the quotation above: 'Immunogenetics is where at least some of medicine's fundamental issues will be decided'.

R. HARRIS

\section{Developmental Biology and Pathology. (Current Topics in Pathology.)}

Edited by A. Gropp and K. Benirschke. (Pp. 208; 86 Figures. DM. 96; \$39.40.) Berlin, Heidelberg, New York: Springer-Verlag. 1976.

This volume was conceived at a Symposium on 'Control of Early Embryogenesis and Factors Responsible for Failure of Embryonic Development', held from 1 to 4 May 1974 in Travemunde, and sponsored by the Deutsche Forschungsgemeinschaft. It consists of a collection of 14 of the papers presented, and includes an article on 'Oocyte Maturation and Paternal Contribution to the Embryo in Mammals' by D. Szöllösi, a concise and heavily referenced review of a large amount of highly technical data. It also includes an article on 'Maternal Storage in the Mammalian Oocyte' by W. Engel and W. Franke, which reveals the gaps in our knowledge, and the marked species variation concerning RNA protein synthesis in the developing and fertilised oocyte. A paper by H. W. Denker on 'Formation of the Blastocyst ...' provides an interesting review of the theories regarding differentiation, and one by Cecilia LutwakMann on 'The Response of the Preimplantation Embryo to Exogenous Factors' re-emphasises the resilience of the embryo at that early stage. 'Embryo Transfer Technique and Action of Drugs on the Preimplantation Embryo' by H. Spielmann highlights the methodological shortcomings, and an article on 'Uterine Secretion Protein Patterns Under Hormonal Influences' by $\mathbf{H}$. M. Beier reviews levels of progesterone and oestrogens in pregnant and pseudopregnant experiment animals. An article on 'Organ Culture in Teratology' by L. Saxén and his coworkers reveals the technique of organ culture as used to define the mechanisms in teratogenesis. Three papers on chromosomal aspects, 'Experimental Heteroploidy in Mammals' by $\mathrm{O}$. Bomsel-Helmreich, 'Autosomal Monosomy and Trisomy Causing Developmental Failure' by A. Gropp and his coworkers, and 'Chromosomal Abnormalities in Early Abortions' by J. G. Boué and A. Boué, form a useful review.

These reviews are generally heavily referenced and several contain details of the individual author's work, but no summary of the discussion which must surely have followed. However, this published symposium, in common with so many others, provides too little experimental detail for the individual working in this field, and not enough introductory and explanatory material for the new investigator or for those interested in bringing their knowledge regarding embryogenesis and embryopathy up to date. However, the number of referenced papers ought to provide good source material. Once criticism is of the editorial construction, particularly with the careless labelling of essential illustrations. For example, in labelling electron photomicrographs, the text refers to cortical granules abbreviated CG, while the photographs of the granules appear quoted as GC. In conclusion, this is a collection of interesting papers, which might well have been better published in a journal where they would receive wider dissemination to those specifically interested in the general topic.

K. M. LAURENCE

\section{Le Polymorphisme Biochimique et Les Facteurs de Son Maintien \\ By Gérard Lucotte. (Pp. 59; Figures + Tables. $\$ 12 \cdot 74$.$) Paris: Masson. 1977$.}

This clear and manageable little book will be useful and interesting both to students (French speaking) and a wider audience. It is a concise summary of the literature on the selective mechanisms which may be involved in the maintenance of the so-called biochemical polymorphisms. The 'neutralist' hypothesis, which implicates random genetic drift rather than selection, is also covered, but is unfortunately less well explained, and one suspects this reflects the author's selectionist bias.

Each chapter is accompanied by an extensive bibliography, each reference with a full title. The lists of references are also subdivided under headings, so that the reader is given extra information about the content of the articles. It is, however, a great pity that the references mentioned in the text do not always appear in the bibliographies.

The first 2 chapters deal with the nature and extent of 'biochemical' polymorphisms, as detectable by 